\title{
A 3-Subset Meet-in-the-Middle Attack: Cryptanalysis of the Lightweight Block Cipher KTANTAN
}

\author{
Andrey Bogdanov and Christian Rechberger \\ Katholieke Universiteit Leuven, ESAT/COSIC and IBBT, Belgium \\ \{andrey.bogdanov, christian.rechberber\}@esat.kuleuven.be
}

\begin{abstract}
In this paper we describe a variant of existing meet-in-themiddle attacks on block ciphers. As an application, we propose meetin-the-middle attacks that are applicable to the KTANTAN family of block ciphers accepting a key of 80 bits. The attacks are due to some weaknesses in its bitwise key schedule1. We report an attack of time complexity $2^{75.170}$ encryptions on the full KTANTAN32 cipher with only 3 plaintext/ciphertext pairs and well as $2^{75.044}$ encryptions on the full KTANTAN48 and $2^{75.584}$ encryptions on the full KTANTAN64 with 2 plaintext/ciphertext pairs. All these attacks work in the classical attack model without any related keys.

In the differential related-key model, we demonstrate 218- and 174round differentials holding with probability 1 . This shows that a strong related-key property can translate to a successful attack in the nonrelated-key setting. Having extremely low data requirements, these attacks are valid even in RFID-like environments where only a very limited amount of text material may be available to an attacker.
\end{abstract}

Keywords: cryptanalysis, meet-in-the-middle attacks, block cipher, key schedule, lightweight cipher, key-recovery, RFID.

\section{Introduction}

A number of new cipher designs have been proposed recently, targeting use cases with severe implementation constraints imposed. Block cipher design methods have advanced to a stage where strong arguments for the resistance of the design against large classes of attacks such as differential and linear cryptanalysis are possible. However, if aggressive design decisions have been made forced by a restrictive application scenario, some other, more dedicated analysis techniques may turn out useful for attacking the cipher.

Cryptographic techniques move into applications like sensor nodes, RFID tags, or the "the Internet of things" at large. The ever increasing demand for security

${ }^{1}$ The SAC 2010 pre-proceedings version of this paper [6] was based on a key-schedule from a previous version of the reference code which contained errors. This paper is based on the corrected reference code available under [1].

A. Biryukov, G. Gong, and D.R. Stinson (Eds.): SAC 2010, LNCS 6544, pp. 229 240, 2011.

(C) Springer-Verlag Berlin Heidelberg 2011 
and privacy in these very constrained environments requires new cryptographic primitives, like tiny yet efficient ciphers. A number of designs and implementation techniques have been proposed recently to address this need. Stream ciphers like Trivium [108], Grain [1718, or Mickey [3], or block ciphers like DESL [22], PRESENT [4, HIGHT [19, mCrypton [23, KATAN and KTANTAN 9], or the hash functions based on PRESENT [5] are among the important ones.

Motivation. The economical and physical constraints force designers to make design decisions which are often considered to be "on the edge". In this context it is often argued that block ciphers are better understood than stream ciphers and are hence more trustworthy. Some recent designs like PRESENT and KATAN/KTANTAN have come with strong arguments that large classes of attacks shown powerful in the past are not applicable. The technique used is to provide bounds on various non-random properties, like differential or linear characteristics. Indeed, whereas in the eStream project a number of lightweight stream ciphers were broken, sometimes even with practical attack complexities, none of the recently proposed block ciphers have been broken so far.

KTANTAN [9] accepts a key of 80 bits. It was designed to resist differential and linear attacks, and exhibits strong bounds in the non-related-key model, an upper bound $2^{-b}$ on the probability of every differential/linear characteristic over 128 rounds being an essential design criterion $(b \in\{32,48,64\}$ is the block size of the cipher). Even if related keys are considered, a much less realistic setting, designers report no differential characteristic with a higher probability than $2^{-b}$ for 150 out of the 254 rounds. In [2], Albrecht et al. study algebraic approaches to amplify differential attacks on this family of ciphers.

Contributions and Outline. Section 2 considers a framework for MITM attacks. In Section 4, based on this framework, we propose a key-recovery attack on the KTANTAN block cipher family (briefly described in Section 3), requiring only very few known plaintext/ciphertext pairs. Hence this kind of attacks is even valid in very restrictive RFID-like environments and protocols where only a very limited number of transactions are foreseen in the lifetime of a tag. The parameters and complexities of our attacks are provided in Table 1. Some properties we use translate to probability-1 related-key differentials over many rounds which are outlined in Table 4. Note that the data complexity of our attacks is

Table 1. Results on MITM cryptanalysis for KTANTAN

\begin{tabular}{l|cccc}
\hline attack/bound & $\begin{array}{c}\text { cipher } \\
\text { \# }\end{array}$ & $\begin{array}{c}\text { \#rounds } \\
\text { (of 254) }\end{array}$ & $\begin{array}{c}\text { time } \\
\text { [encryptions] }\end{array}$ & $\begin{array}{c}\text { data compl. } \\
{[\text { PT/CT pars }]}\end{array}$ \\
\hline \hline$\underline{9}$, RK diff. bound & KTANTANb & 150 & $\mathcal{O}\left(2^{b}\right)$ & $\mathcal{O}\left(2^{b}\right)$ \\
9], DC and LC bound & KTANTANb & 128 & $\mathcal{O}\left(2^{b}\right)$ & $\mathcal{O}\left(2^{b}\right)$ \\
\hline this paper, MITM attack & KTANTAN32 & 254 & $2^{75.170}$ & 3 \\
this paper, MITM attack & KTANTAN48 & 254 & $2^{75.044}$ & 2 \\
this paper, MITM attack & KTANTAN64 & 254 & $2^{75.584}$ & 2
\end{tabular}


the lowest possible and exactly corresponds to that of a brute-force attack. We conclude with a discussion on links to other works, high-level design choices for low-resource ciphers, and future work in Section 5 .

\section{Framework for MITM Attacks}

\subsection{Basic MITM Attack}

The basic meet-in-the-middle (MITM) approach will be a starting point for our attack. MITM techniques are arguably much less common than differential or linear attacks on ciphers. There are some applications of MITM principles to block ciphers like DES or AES, see e.g. 7/11/12 14 15|20] for dedicated attacks, and e.g. 24 25] for meet-in-the-middle attacks on a higher level.

The basic MITM technique is due to Diffie and Hellman [13. Let $\varphi_{i, j}$ denote the partial transform of an $R$-round block cipher beginning in round $i$ and ending directly after round $j$ under some fixed key, $1 \leq i \leq j \leq R$, see Figure 1. Then if $\varphi_{1, \alpha}$ and $\varphi_{\alpha+1, R}$ use subkeys with distinct key bits, the key can be as a rule recovered much more efficiently than by brute force over two subkeys. The central idea here, as also applied to reduced DES in [15], is that the subkeys in both parts of the cipher can be guessed independently. Each guess of the first subkey allows the adversary to compute $\varphi_{1, \alpha}(p)$ and of the second subkey to obtain $\varphi_{\alpha+1, R}^{-1}(c)$. The right key will be among those fulfilling the equation $\varphi_{1, \alpha}(p)=\varphi_{\alpha+1, R}^{-1}(c)$.

\subsection{The 3-Subset MITM Approach}

Here we consider a variant of the basic MITM attack. The idea is to remove restrictions on the choice of key bits, thereby potentially allowing attacks where an attack is not possible with the basic MITM approach. Instead of considering two subsets of key bits, we consider three subsets. The attack consists of two parts. In the MITM stage, we filter out some wrong key candidates and reduce the key space. In the key testing stage, we look for the right key in the reduced key space.

Let $K=k_{\ell-1} k_{\ell-2} \ldots k_{1} k_{0}$ be the $\ell$-bit key. Then if $K_{1}=\left\{k_{i}: k_{i}\right.$ used by $\left.\varphi_{1, \alpha}\right\}$ and $K_{2}=\left\{k_{i}: k_{i}\right.$ used by $\left.\varphi_{R-\beta+1, R}\right\}$, then $A_{0}=K_{1} \cap K_{2}$ is the set of key

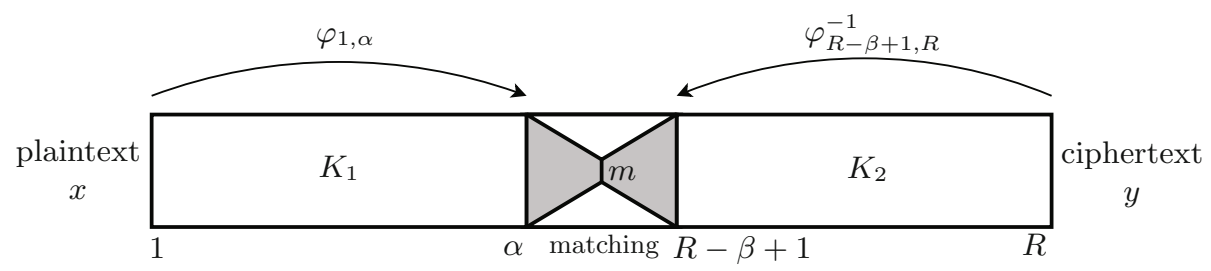

Fig. 1. MITM 
bits used both by the first $\alpha$ and last $\beta$ rounds, see Figure 1. Moreover, $A_{1}=$ $K_{1} \backslash K_{1} \cap K_{2}$ and $A_{2}=K_{2} \backslash K_{1} \cap K_{2}$ are the sets of key bits used by $K_{1}$ only and by $K_{2}$ only, respectively. We further assume that $K_{1} \cup K_{2}=K$.

For the attack we need $n$ plaintext/ciphertext pairs $\left\{\left(x_{i}, y_{i}\right)\right\}, i=1, \ldots, n$. Let $x_{i}$ be plaintext and $y_{i}$ ciphertext.

MITM Stage. The meet-in-the-middle part of the attack on $R$ rounds of the cipher can be performed as follows:

- For each guess of key bits in $A_{0}$ :

- For each guess of key bits in $A_{1}$ :

* Compute $v=\varphi_{1, \alpha}(x)$

- For each guess of key bits in $A_{2}$ :

* Compute $u=\varphi_{R-\beta+1, R}^{-1}(y)$

- Perform matching in the middle between the values of $v$ and the values of $u$ on $m$ bits, $1 \leq m \leq b$ (see Subsection 4.3) and add surviving key candidates to the list $\mathcal{K}$ of surviving keys. We expect to have false positives with probability $2^{-m}$ which is called the false positive rate of a MITM attack.

Key Testing Stage. In this stage, we test the surviving key candidates from $\mathcal{K}$ using some plaintext-ciphertext pairs in a brute-force manner. Generally speaking, it is not necessary to use additional plaintext-ciphertext pairs. The number of the texts needed is defined by the unicity distance of the cipher which essentially depends on the block size and key length. If we can significantly reduce the key space in the MITM stage (by ruling out a large part of the keys), the complexity of the key testing stage will be negligible with respect to the MITM stage. Generally speaking, however, this is not necessarily the case.

Attack Complexity. The computational complexity of the attack will be dominated by

$$
C_{\text {comp }}=\underbrace{2^{\left|A_{0}\right|}\left(2^{\left|A_{1}\right|}+2^{\left|A_{2}\right|}\right)}_{\text {MITM stage }}+\underbrace{\left(2^{\ell-m}+2^{\ell-m-b}+2^{\ell-m-2 b}+\ldots\right)}_{\text {key testing stage }} .
$$

If $A_{1}$ and $A_{2}$ are both non-empty and $\left|A_{1}\right|+\left|A_{2}\right|>2$, then the attack becomes more efficient than exhaustive search provided that the false positive rate is low enough.

The MITM stage requires exactly one plaintext/ciphertext pair. However, of the $b$ bits only $m$ are used for matching. That is, the information contained in the other $b-m$ state bits is not used in this stage and can be used in the key testing stage. For the key testing stage, more pairs might be required, depending on the relation between the key length and the block size. This results in data complexity

$$
C_{\text {data }}=\left\lceil\frac{\ell}{b}\right\rceil
$$

depending on the block size $b$ and the key length $\ell$. The memory complexity is defined by matching in the MITM stage. For small sets $A_{1}$ and $A_{2}$, it is negligible. 


\section{A Short Description of KTANTAN}

KTANTAN is a block cipher which accepts an 80-bit user-supplied key. Versions with block size $b \in\{32,48,64\}$ bit have been specified. Each version has 254 rounds. While the definition of a round transform differs from version to version, the key schedule remains the same. Throughout the paper, we refer to the KTANTAN version with $b$-bit blocks as KTANTANb.

\subsection{Round Transform}

KATAN and KTANTAN share the specification of a round transform, as the operations on the state are exactly the same up to the key schedule. The state of the cipher is represented as two disjunct parts $L_{1}$ and $L_{2}$. The transform of round $r$ is based on two Boolean functions $f_{1, r}$ and $f_{2, r}$, having $L_{1}$ and $L_{2}$ as their domain, correspondingly:

$$
\begin{aligned}
& f_{1, r}\left(L_{1}\right)=L_{1}\left[x_{1}\right] \oplus L_{1}\left[x_{2}\right] \oplus\left(L_{1}\left[x_{3}\right] \cdot L_{1}\left[x_{4}\right]\right) \oplus\left(L_{1}\left[x_{5}\right] \cdot I R_{r}\right) \oplus \kappa_{1, r} \\
& f_{2, r}\left(L_{2}\right)=L_{2}\left[y_{1}\right] \oplus L_{2}\left[y_{2}\right] \oplus\left(L_{2}\left[y_{3}\right] \cdot L_{2}\left[y_{4}\right]\right) \oplus\left(L_{2}\left[y_{5}\right] \cdot L_{2}\left[y_{6}\right]\right) \oplus \kappa_{2, r},
\end{aligned}
$$

where $x_{i}, y_{i}$ are the numbers of active bit positions, $I R_{r}$ is the round constant bit in round $r$, and $\kappa_{1, r}, \kappa_{2, r}$ are the bits of the extended key defined by the key schedule for round $r$. The lengths of $L_{1}$ and $L_{2}$ as well as the bit positions $x_{i}$, $y_{i}$ are specific for each KTANTAN version.

Once $f_{1, r}$ and $f_{2, r}$ are computed, the registers $L_{1}$ and $L_{2}$ are shifted, the MSB of each register falls off and the LSB is set to the output of $f_{2, r}$ and the output of $f_{1, r}$, respectively. KTANTAN32 applies transformations $f_{1, r}$ and $f_{2, r}$ once in a round. One round of KTANTAN48 and KTANTAN64 updates the registers using $f_{1, r}$ and $f_{2, r}$ two and three times, respectively.

Table 2. Version-specific parameters of KTANTAN

\begin{tabular}{c|c|ccccccccccccc}
$b$ & $\left|L_{1}\right|$ & $L_{2} \mid$ & $x_{1}$ & $x_{2}$ & $x_{3}$ & $x_{4}$ & $x_{5}$ & $y_{1}$ & $y_{2}$ & $y_{3}$ & $y_{4}$ & $y_{5}$ & $y_{6}$ \\
\hline \hline 32 & 13 & 19 & 12 & 7 & 8 & 5 & 3 & 18 & 7 & 12 & 10 & 8 & 3 \\
48 & 19 & 29 & 18 & 12 & 15 & 7 & 6 & 28 & 19 & 21 & 13 & 15 & 6 \\
64 & 25 & 39 & 24 & 15 & 20 & 11 & 9 & 38 & 25 & 33 & 21 & 14 & 9
\end{tabular}

Table 3. Round constant bits $I R_{r}: I R_{1}$ first, $I R_{254}$ last

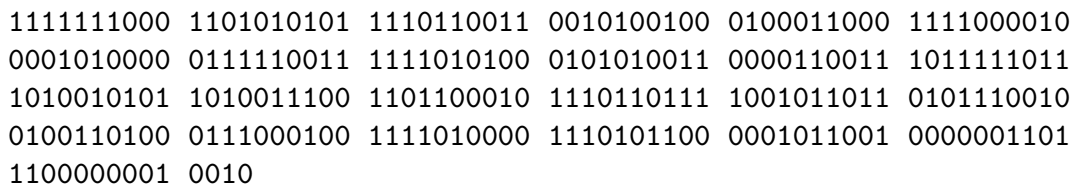




\subsection{Key Schedule}

The functions $f_{1, r}$ and $f_{2, r}$ require input from the key schedule, which is a function mapping the 80-bit user-supplied key $K=k_{79} k_{78} \ldots k_{1} k_{0}$ to $\kappa_{1, r}$ and $\kappa_{2, r}$ for each round $r$. It is exactly this part where KTANTAN differs from KATAN. This difference, together with some properties of the data transform, makes KTANTAN vulnerable to our attack.

An 8-bit round counting LFSR is used to control the key schedule. It is defined by the feedback polynomial

$$
\zeta^{8}+\zeta^{7}+\zeta^{5}+\zeta^{3}+1
$$

and its initial state is all ones. The value of $I R_{r}$ is specified as the most significant bit of this LFSR in round $r$. Let $l_{7, r} l_{6, r} \ldots l_{1, r} l_{0, r}$ denote the 8 -bit state of the LFSR in round $r$.

The key schedule of KTANTAN chooses two bits of $K$ in each round. This is done by applying two layers of MUX logic. First, $K$ is divided into 5 chunks $W_{i}$ of 16 bits each: $K=W_{4}|| W_{3}\left\|W_{2}\right\| W_{1} \| W_{0}$. One bit out of each chunk is selected:

$$
\omega_{i, r}=\operatorname{MUX16to1}\left(W_{i}, l_{7, r} l_{6, r} l_{5, r} l_{4, r}\right), i=0, \ldots, 4,
$$

where the LFSR bits define the position in $W_{i}$ to choose. Second, two out of these five bits are chosen controlled by the other half of the LFSR state:

$$
\begin{aligned}
& \kappa_{1, r}=\overline{l_{3, r}} \cdot \overline{l_{2, r}} \cdot \omega_{0, r} \oplus\left(l_{3, r} \vee l_{2, r}\right) \cdot \operatorname{MUX} 4 \operatorname{to1}\left(\omega_{4, r} \omega_{3, r} \omega_{2, r} \omega_{1, r}, l_{1, r} l_{0, r}\right) \\
& \kappa_{2, r}=\overline{l_{3, r}} \cdot l_{2, r} \cdot \omega_{4, r} \oplus\left(l_{3, r} \vee \overline{l_{2, r}}\right) \cdot \operatorname{MUX4to1}\left(\omega_{3, r} \omega_{2, r} \omega_{1, r} \omega_{0, r}, l_{1, r} l_{0, r}\right) .
\end{aligned}
$$

\section{Low Data-Complexity Attacks on KTANTAN}

In here, we apply the MITM framework described in Section 2 to all 3 variants of the full KTANTAN with 254 rounds. The resulting attack requires an extremely small number of known plaintext/ciphertext pairs (basically, the minimum due to the unicity distance) and negligible memory. The MITM techniques make use of the fact that several key bits remain unused by the KTANTAN key schedule in large connected parts of the cipher. More precisely, it is the rounds at the beginning and end of KTANTAN which we are most interested in to make the attack work.

\subsection{Related-Key Differentials of Probability 1}

We start with a note on probability-1 related-key differentials of KTANTAN over many rounds.

The key observation here is that if certain key bits are not used over many rounds, they can be flipped without affecting the data transformation. In other words, the $R$-round related-key differential $(0, \Delta) \mapsto 0$ holds with probability 1 , where 0 is the zero input and output difference in the data transformation and $\Delta$ is the key difference with ones at key bit position not used in the $R$ rounds 
Table 4. Related key differentials for KTANTAN $b, b \in\{32,48,64\}$

\begin{tabular}{l|c|c|c}
\hline $\begin{array}{c}\text { covered } \\
\text { rounds }\end{array}$ & \#rounds & differential & probability \\
\hline \hline$\varphi_{1,218}$ & 218 & $(0,00000000800000000000) \mapsto 0$ & 1 \\
$\varphi_{81,254}^{-1}$ & 174 & $(0,00000000000000010000) \mapsto 0$ & 1
\end{tabular}

(first key bit positions are on the left-hand side). Some of the longest relatedkey differentials of this type we found for KTANTAN $b$ are given in Table 4 . These differentials are due to the fact that the first 218 and last 174 rounds of KTANTAN do not use key bits $k_{32}$ and $k_{63}$, respectively.

We notice that it seems possible that these properties can be turned into low-complexity differential related-key attacks on KTANTAN. However, we are mostly concerned with attacks that do not require related keys, and hence continue by exploiting this property in another way.

\subsection{Application of the MITM Framework to KTANTAN}

Depending on the KTANTAN version, different properties of the key schedule are exploited in our attack. This is due to the fact that all three versions of KTANTAN have different numbers of register clocks in one round. The versions with a larger block size have heavier rounds with more diffusion which complicates the partial matching phase. Effectively, this might reduce the number of rounds in the middle for which matching is possible.

In Table 5 we give a summary of the properties and parameters of our attacks. We aim for the full, and if this is not possible for the highest number of rounds. Considering variants with a more reduced number of rounds would lead to more neutrals key bits, and generally lower attack complexities.

To illustrate the meaning of this table, let us consider the first entry. We attack the full 254-round KTANTAN32. The two basic properties of the KTANTAN key schedule which make our attack on KTANTAN32 possible can be formulated as:

Fact 1. $\varphi_{1, \alpha}$ does not use key bits $\left\{k_{32}, k_{39}, k_{44}, k_{61}, k_{66}, k_{75}\right\}$ for $1 \leq \alpha \leq 111$.

Fact 2. $\varphi_{254-\beta+1,254}$ does not use key bits $\left\{k_{3}, k_{20}, k_{41}, k_{47}, k_{63}, k_{74}\right\}$ for $1 \leq$ $\beta \leq 131$.

Table 5. Details of the proposed attacks

\begin{tabular}{c|c||c|c||c|c||c||c|c|c}
$b$ & $R$ & $\alpha$ & $A_{1}$ & $R-\beta$ & $A_{2}$ & matching & \multicolumn{3}{|c}{ complexity $C_{\text {comp }}$} \\
& & & & & & bits $m$ & MITM & key test & total \\
\hline \hline 32 & 254 & 111 & $32,39,44,61,66,75$ & 131 & $3,20,41,47,63,74$ & 8 & 75.000 & 72 & 75.170 \\
48 & 254 & 111 & $32,39,44,61,66,75$ & 131 & $3,20,41,47,63,74$ & 10 & 75.000 & 70 & 75.044 \\
64 & 254 & 123 & $32,44,61,66,75$ & 131 & $3,20,41,47,63,74$ & 47 & 75.584 & 33 & 75.584
\end{tabular}


This means that if we fix $\alpha=111$ and $\beta=131$, then $\varphi_{1,111}$ and $\varphi_{123,254}$ will have 6 neutral key bits in each direction. Moreover, one can efficiently match $v$ and $u$ in 8 bits, despite being 21 rounds apart due to the slow diffusion in one round of KTANTAN32, which is demonstrated in Subsection 4.3.

\subsection{Partial-Matching Phase}

In here, we describe in more detail the matching procedure that is used as a sub-routine in the MITM stage.

Procedure. The starting point are two completely determined internal states $u$ and $v$ several rounds apart (for KTANTAN32, one at round 111 and the other one at round 131). If we considered a cipher where those middle rounds were cut away, then the matching phase would be trivial as we would simply check if the $u=v$. With a probability of about $2^{-b}$ this check would give a false positive, but overall the number of key candidates is reduced to about $2^{80-b}$. Remember that $b \in\{32,48,64\}$. Hence the number of key candidates is small enough to not influence the attack complexity during the key testing state.

In order to bridge this gap and to obtain a result on the full cipher, we drop the requirement to match on every state bit but allow for a much smaller number of matched bits $m$. This will increase the number of false positives, but in a way that does not noticeably influence any property of the attack. In more detail, we find that $m$ bits (for KTANTAN32, $m=8$ ) will still match with probability 1 (see below for details). This means that we will have reduced the number of key candidates to $2^{80-m}$ after the MITM stage. In total, we hence need only between 2 (for block size $b=\{48,64\}$ ) or 3 (for $b=32$ ) known plaintext/ciphertext pairs.

We note that this procedure can be implemented in an essentially memoryless way, as every match can immediately be tested with another plaintext/ciphertext pair. Also in our estimate of the attack complexity, we do not consider any implementation optimizations that e.g. would also be possible for a brute force search that does not use any shortcut attacks. Examples of such optimizations would e.g. be a reuse of computations from one key guess to the next.

Details on the Partial Matching Phase. In the following we trace those bits that remain unaffected during the middle rounds for the block size of $b=32$. ' 1 ' means affected, '0' means not affected. $k 1$ and $k 2$ denote disturbances caused by the unknown neutral bits from the opposite chunk at the respective rounds. For other block sizes, we refer to Appendix A. 


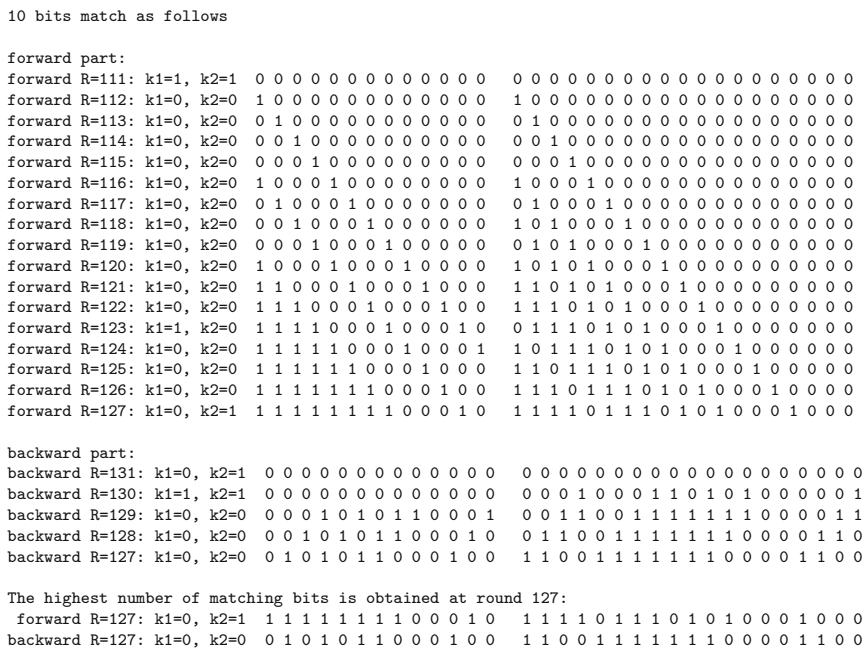

As can be seen, at three positions in the state both in the forward and in the backward part no changes happen, which is the property needed for the matching part.

\section{Discussion and Future Work}

The recently proposed lightweight block cipher KTANTAN is susceptible to a class of meet-in-the-middle attacks that seems to put less constraints on the selection of key bits than some earlier meet-in-the-middle attacks on block ciphers. We proposed key-recovery attacks with an extremely low number of known plaintexts. The approach we describe is inspired by recent advances in MITM preimage attacks on hash functions like those that succeeded in breaking MD5 or Tiger [16 27], even though it remains an open problem to transfer most of the techniques there from the key-less hash setting to the cipher setting. The MITM approach may be seen as a way to turn very strong related-key properties into attacks in the single-key setting, complementing e.g. the work on the selfsynchronized stream-cipher Moustique [21]. Even though the time complexity of our attack remains high, optimizations may result in reduced time complexities, by e.g. allowing the attacker to choose the plaintext instead (possible in many protocols), or asking for more plaintext/ciphertext pairs. Also, implementation techniques that speed-up brute force search, such as determining a good sequence of keys to guess and save computations that way, are likely to carry over to the meet-in-the-middle attack.

Among the ciphers most vulnerable to the meet-in-the-middle attacks, are those with little key-dependency in the sense that large parts of the cipher depend on a subset of key bits only. This is opposed to substitution-permutation networks which usually use subkeys of the block length in each round. For such ciphers, it is often difficult to mount a meet-in-the-middle attack even on a small number of rounds because of the strong key dependency. However, this approach 
as a rule results in a much higher number of XOR-operations needed for the key addition which in turn leads to higher area and/or time requirements and, thus, to a lower efficiency. An optimal trade-off between the level of resistance and the amount of key dependency remains, however, an area of research.

Acknowledgements. Andrey Bogdanov was supported in part by a visiting postdoctoral fellow grant from the Fund for Scientific Research - Flanders (FWO) within the FWO research project "Linear codes and cryptography" G.0317.06. This work was also sponsored by the Research Fund K.U.Leuven grant (OT/08/027) "A mathematical theory for the design of symmetric primitives", by the IAP Programme P6/26 BCRYPT of the Belgian State (Belgian Science Policy), and by the European Commission under contract ICT-2007216646 (ECRYPT II).

The authors are grateful to the designers of KATAN/KTANTAN for clarifying the issues with the KTANTAN key schedule and would like to thank the anonymous reviewers of SAC 2010 whose insightful comments improved the presentation of the paper.

\section{References}

1. Bit-sliced reference code of KATAN and KTANTAN (2010), http://www.cs.technion.ac.il/ orrd/KATAN/katan.c

2. Albrecht, M., Cid, C., Dullien, T., Faugre, J.C., Perret, L.: Algebraic Precomputations in Differential Cryptanalysis. In: ECRYPT Tools for Cryptanalysis Workshop $2010(2010)$

3. Babbage, S., Dodd, M.: The MICKEY Stream Ciphers. In: Robshaw and Billet [26], pp. 191-209

4. Bogdanov, A., Knudsen, L.R., Leander, G., Paar, C., Poschmann, A., Robshaw, M.J.B., Seurin, Y., Vikkelsoe, C.: PRESENT: An Ultra-Lightweight Block Cipher. In: Paillier, P., Verbauwhede, I. (eds.) CHES 2007. LNCS, vol. 4727, pp. 450-466. Springer, Heidelberg (2007)

5. Bogdanov, A., Leander, G., Paar, C., Poschmann, A., Robshaw, M.J.B., Seurin, Y.: Hash Functions and RFID Tags: Mind the Gap. In: Oswald, E., Rohatgi, P. (eds.) CHES 2008. LNCS, vol. 5154, pp. 283-299. Springer, Heidelberg (2008)

6. Bogdanov, A., Rechberger, C.: Generalized Meet-in-the-Middle Attacks: Cryptanalysis of the Lightweight Block Cipher KTANTAN. In: Biryukov, A., Gong, G., Stinson, D.R. (eds.) SAC 2010. LNCS, vol. 6544. Springer, Heidelberg (2010)

7. Chaum, D., Evertse, J.H.: Cryptanalysis of DES with a Reduced Number of Rounds. In: Williams, H.C. (ed.) CRYPTO 1985. LNCS, vol. 218, pp. 192-211. Springer, Heidelberg (1986)

8. De Cannière, C.: Trivium: A Stream Cipher Construction Inspired by Block Cipher Design Principles. In: Katsikas, S.K., López, J., Backes, M., Gritzalis, S., Preneel, B. (eds.) ISC 2006. LNCS, vol. 4176, pp. 171-186. Springer, Heidelberg (2006)

9. De Cannière, C., Dunkelman, O., Knezevic, M.: KATAN and KTANTAN - A Family of Small and Efficient Hardware-Oriented Block Ciphers. In: Clavier, C., Gaj, K. (eds.) CHES 2009. LNCS, vol. 5747, pp. 272-288. Springer, Heidelberg (2009) 
10. De Cannière, C., Preneel, B.: Trivium. In: Robshaw and Billet [26], pp. 244-266

11. Demirci, H., Selçuk, A.A.: A Meet-in-the-Middle Attack on 8-Round AES. In: Nyberg, K. (ed.) FSE 2008. LNCS, vol. 5086, pp. 116-126. Springer, Heidelberg (2008)

12. Demirci, H., Taskin, I., Çoban, M., Baysal, A.: Improved Meet-in-the-Middle Attacks on AES. In: Roy, B., Sendrier, N. (eds.) INDOCRYPT 2009. LNCS, vol. 5922, pp. 144-156. Springer, Heidelberg (2009)

13. Diffie, W., Hellman, M.: Exhaustive Cryptanalysis of the NBS Data Encryption standard. Computer 10(6), 74-84 (1977)

14. Dunkelman, O., Keller, N., Shamir, A.: Improved Single-Key Attacks on 8-round AES. Cryptology ePrint Archive, Report 2010/322 (2010), http://eprint.iacr.org/

15. Dunkelman, O., Sekar, G., Preneel, B.: Improved Meet-in-the-Middle Attacks on Reduced-Round DES. In: Srinathan, K., Rangan, C.P., Yung, M. (eds.) INDOCRYPT 2007. LNCS, vol. 4859, pp. 86-100. Springer, Heidelberg (2007)

16. Guo, J., Ling, S., Rechberger, C., Wang, H.: Advanced Meet-in-the-Middle Preimage Attacks: First Results on Full Tiger, and Improved Results on MD4 and SHA-2. Cryptology ePrint Archive, Report 2010/016 (2010), http://eprint.iacr.org/

17. Hell, M., Johansson, T., Maximov, A., Meier, W.: The Grain Family of Stream Ciphers. In: Robshaw and Billet [26], pp. 179-190

18. Hell, M., Johansson, T., Meier, W.: Grain: a stream cipher for constrained environments. IJWMC 2(1), 86-93 (2007)

19. Hong, D., Sung, J., Hong, S., Lim, J., Lee, S., Koo, B., Lee, C., Chang, D., Lee, J., Jeong, K., Kim, H., Kim, J., Chee, S.: HIGHT: A New Block Cipher Suitable for Low-Resource Device. In: Goubin, L., Matsui, M. (eds.) CHES 2006. LNCS, vol. 4249, pp. 46-59. Springer, Heidelberg (2006)

20. Indesteege, S., Keller, N., Dunkelman, O., Biham, E., Preneel, B.: A Practical Attack on KeeLoq. In: Smart, N.P. (ed.) EUROCRYPT 2008. LNCS, vol. 4965, pp. 1-18. Springer, Heidelberg (2008)

21. Käsper, E., Rijmen, V., Bjørstad, T.E., Rechberger, C., Robshaw, M.J.B., Sekar, G.: Correlated Keystreams in Moustique. In: Vaudenay, S. (ed.) AFRICACRYPT 2008. LNCS, vol. 5023, pp. 246-257. Springer, Heidelberg (2008)

22. Leander, G., Paar, C., Poschmann, A., Schramm, K.: New Lightweight DES Variants. In: Biryukov, A. (ed.) FSE 2007. LNCS, vol. 4593, pp. 196-210. Springer, Heidelberg (2007)

23. Lim, C.H., Korkishko, T.: mCrypton - A Lightweight Block Cipher for Security of Low-Cost RFID Tags and Sensors. In: Song, J., Kwon, T., Yung, M. (eds.) WISA 2005. LNCS, vol. 3786, pp. 243-258. Springer, Heidelberg (2006)

24. Merkle, R.C., Hellman, M.E.: On the Security of Multiple Encryption. Commun. ACM 24(7), 465-467 (1981)

25. van Oorschot, P.C., Wiener, M.J.: A Known-Plaintext Attack on Two-Key Triple Encryption. In: Damgård, I.B. (ed.) EUROCRYPT 1990. LNCS, vol. 473, pp. 318 325. Springer, Heidelberg (1991)

26. Robshaw, M.J.B., Billet, O. (eds.): New Stream Cipher Designs. LNCS, vol. 4986. Springer, Heidelberg (2008)

27. Sasaki, Y., Aoki, K.: Finding Preimages in Full MD5 Faster Than Exhaustive Search. In: Joux, A. (ed.) EUROCRYPT 2009. LNCS, vol. 5479, pp. 134-152. Springer, Heidelberg (2009) 


\section{A More Details on Partial Matching}

\section{A.1 Block Size 48 Bits}

The details for the partial matching phase for the attack on KTANTAN with $b=48$ are as follows:

10 bits match as follows

forward part:

forward $R=111: k 1=1, k 2=1$ forward $R=112: k 1=0, k 2=0$ forward $R=113$ : $k 1=0, k 2=0$ forward $R=114: k 1=0, k 2=0$ forward $R=115: k 1=0, k 2=0$ forward $R=116: k 1=0, k 2=0$ forward $R=117: k 1=0, k 2=0$ forward $R=118$ : $k 1=0, k 2=0$ forward $R=119: k 1=0, k 2=0$ forward $R=120$ : $k 1=0, k 2=0$ forward $R=121: k 1=0, k 2=0$ forward $R=122$ : $k 1=0, k 2=0$ forward $R=123: k 1=1, k 2=0$

backward part:

backward $R=131$ : $k 1=0, k 2=1$ backward $\mathrm{R}=130$ : $\mathrm{k} 1=1, \mathrm{k} 2=1$ backward $R=129$ : $k 1=0, k 2=0$ backward $\mathrm{R}=128$ : $\mathrm{k} 1=0, \mathrm{k} 2=0$ backward $R=127$ : $k 1=0, k 2=0$ backward $R=126$ : $k 1=0, k 2=0$ backward $R=125$ : $k 1=0, k 2=0$ backward $R=124$ : $k 1=0, k 2=0$ backward $R=123$ : $k 1=0, k 2=0$
0000000000000000000 1100000000000000000 0011000000000000000 0000110000000000000 1000001100000000000 0110000011000000000 0001100000110000000 0000011000001100000 1100000110000011000 1111000001100000110 0011110000011000001 1100111100000110000 1111001111000001100

0000000000000000000 0000000000000000000 0000011100011011011 0001110001101101100 0111000110110110000 1100011111011011011 0001111101111111111 0111110111111111100 1111011111111111011
00000000000000000000000000000 11000000000000000000000000000 00110000000000000000000000000 00001100000000000000000000000 10000011000000000000000000000 11100000110000000000000000000 00111000001100000000000000000 10001110000011000000000000000 11100011100000110000000000000 11111000111000001100000000000 10111110001110000011000000000 11101111100011100000110000000 11111011111000111000001100000

00000000000000000000000000000 00000110000011110011110000011 00011110001111111111110001111 01111000111111111111000111100 11100011111111111100011110000 10001111111111110001111000000 00111111111111110111110000011 11111111111111011111000001100 11111111111101111100000110000

The highest number of matching bits is obtained at round 123 :

forward R=123: $k 1=1, k 2=0 \quad 111100111100000110011111011111000111000001100000$ backward $\mathrm{R}=123$ : $\mathrm{k} 1=0, \mathrm{k} 2=0 \quad 1111011111111111011 \quad 11111111111101111100000110000$

\section{A.2 Block Size 64 Bits}

The details for the partial matching phase for the attack on KTANTAN with $b=64$ are as follows:

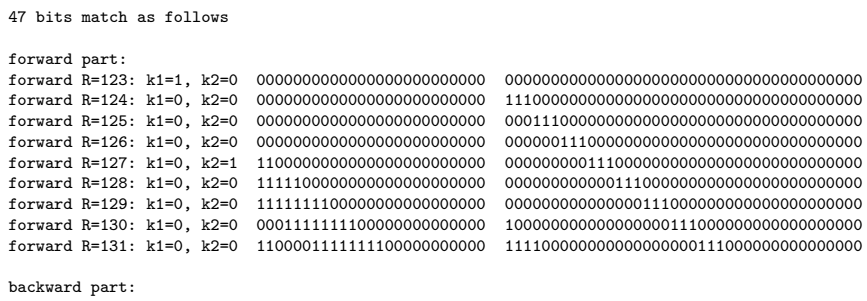

